\title{
Bone marrow suppression as a complication of total skin helical tomotherapy in the treatment of mycosis fungoides
}

\author{
Eric M. Schaff ${ }^{1} \mathbb{D}$, Stephen A. Rosenberg ${ }^{2}$, Stephanie J. Olson², Steven P. Howard ${ }^{2}$ and Kristin A. Bradley²
}

\begin{abstract}
Background: Total skin electron beam therapy (TSEBT) is an effective treatment in mycosis fungoides. Total skin helical tomotherapy (TSHT) may be an alternative to TSEBT and may offer several dosimetric and treatment advantages. There are currently very few published treatment results using TSHT in place of TSEBT for treatment of mycosis fungoides.

Case presentation: Two patients with mycosis fungoides were treated at our institution using TSHT. The first patient was a 69-year-old Caucasian female with stage IVA2 (T2 N3 MO B2) disease who was treated to a dose of 12 Gy in 8 fractions, with a bone marrow mean dose of $1.66 \mathrm{~Gy}$ and $\mathrm{V} 10=0.41 \%$. Two weeks after ending treatment the patient developed myelosuppression including grade 4 thrombocytopenia and required blood and platelet transfusions. The second patient was a 29-year-old Caucasian female with stage I (T2 NO MO BO) disease. This patient previously had been treated for mycosis fungoides using helical tomotherapy $(\mathrm{HT})$ at a dose of $20 \mathrm{~Gy}$ to a localized region and experienced mild thrombocytopenia at that time. The patient then underwent retreatment 17 months later with TSHT to a dose of 12 Gy in 6 fractions with a mean bone marrow dose of $2.3 \mathrm{~Gy}$ and $\mathrm{V} 10=4.28 \%$. This patient once again experienced myelosuppression that included grade 4 thrombocytopenia. She also required blood and platelet transfusions.
\end{abstract}

Conclusions: Both patients treated with TSHT experienced severe bone marrow suppression including grade 4 thrombocytopenia. This was more severe than expected considering the relatively low overall prescription dose and despite a planning constraint placed on the bone marrow of a mean dose of < $2 \mathrm{~Gy}$. These outcomes suggest that patients treated using TSHT should be closely monitored for myelosuppression and caution used even when treating to a dose of $12 \mathrm{~Gy}$.

Keywords: Mycosis fungoides, Helical tomotherapy, Total skin electron beam therapy, Cutaneous lymphoma, T-cell lymphoma

\section{Background}

TSEBT has been proven to be an effective treatment in T2 and T3 mycosis fungoides that is refractory to other treatment modalities. Although it has been proven effective, TSEBT has disadvantages that may complicate treatment [1]. The most common protocol used for treatment is the Stanford Protocol that requires the patient to stand for the entirety of treatment that can last $30 \mathrm{~min}$ [2]. Total skin electron therapy may be limited secondary to heterogeneity with areas receiving up to $40 \%$ deviation from the

\footnotetext{
* Correspondence: kabradley@humonc.wisc.edu

${ }^{2}$ Department of Human Oncology, University of Wisconsin, Madison, WI, USA Full list of author information is available at the end of the article
}

prescription dose [3]. There have been alternative protocols proposed which allow for the patient to lie down but these protocols sacrifice dose homogeneity to an even greater extent [4].

Due to its dose delivery characteristics, helical tomotherapy (HT) with photons can be used to treat superficially and thus may be an alternative to TSEBT. Treating patients with total skin helical tomotherapy (TSHT) may provide several advantages: 1) improved dose homogeneity; 2) non-overlapping fields; and 3) patients in a comfortable supine position for treatment.

Although there are potential benefits to the use of TSHT, there remains concern that photon therapy 

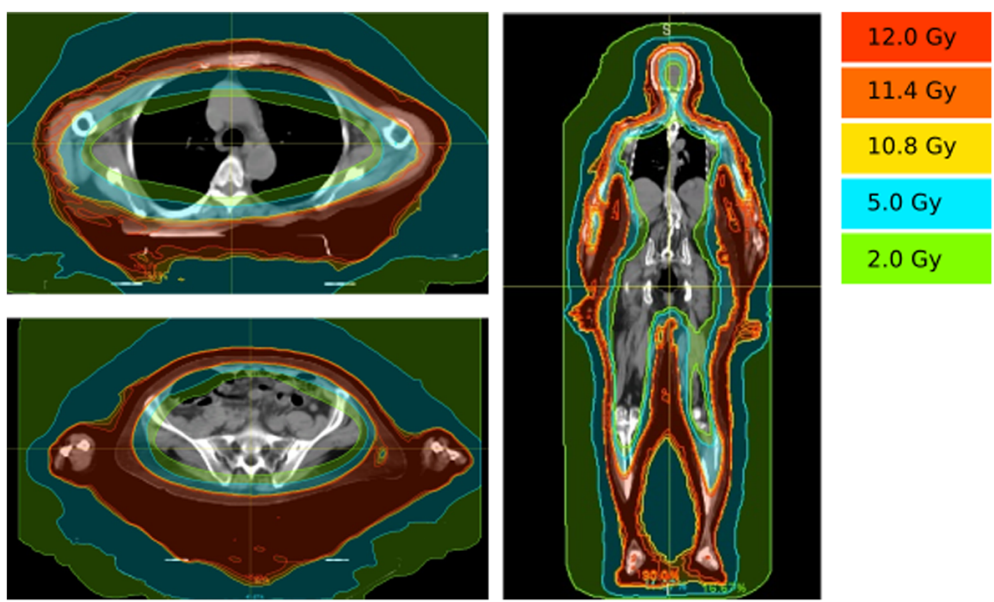

Fig. 1 Dose distributions for patient 1 treated with TSHT for mycosis fungoides

may lead to higher bone marrow doses and potential toxicity compared to TSEBT. Recent data regarding TSEBT suggest that lower doses of radiation may provide equally effective control of the disease, less toxicity, and leave the possibility for retreatment if relapse occurs [5]. A pooled analysis of 3 phase II trials showed an overall response of $88 \%$ and complete response of $27 \%$ treating with 12 Gy utilizing TSEBT [6]. Only $3 \%$ of patients experienced grade 1 leukopenia in these trials. If lower doses are equally effective using TSHT as well, it is possible that the treatment toxicity can be spared while still maintaining treatment efficacy with a photon-based approach. Herein we describe the outcomes of 2 patients treated with TSHT and the toxicity associated with treatment.

\section{Case presentation}

The first patient was a 69-year-old Caucasian female with stage IVA2 (T2 N3 M0 B2) mycosis fungoides who presented to the radiation oncology clinic for treatment after failing interferon alfa-2b, photopheresis, vorinostat, brentuximab vedotin, and gemcitabine. She was treated by our department using TSHT to a dose of 12 Gy in 8 fractions 4 days per week. The patient underwent simulation and treatment with a head and shoulder mask, body fix, with arms down. The dose distribution and dose-volume histogram are shown in Figs. 1 and 2. Bone marrow mean dose was 1.66 Gy with $\mathrm{V} 10=0.41 \%$. The bone marrow contour sum included the entire spine, pelvis and femurs. Dose values to other bony sites have been included for additional reference in Table 1.

On the last day of treatment, she developed grade 1 anemia and thrombocytopenia. Two weeks following the last treatment the bone marrow toxicity progressed to grade 2 anemia, grade 2 leukopenia, and grade 4 thrombocytopenia. The patient was given multiple transfusions of packed red blood cells (PRBCs) and platelets over the course of treatment. One month after treatment she experienced an episode of epistaxis that self-resolved after 40 min. Within 1 week

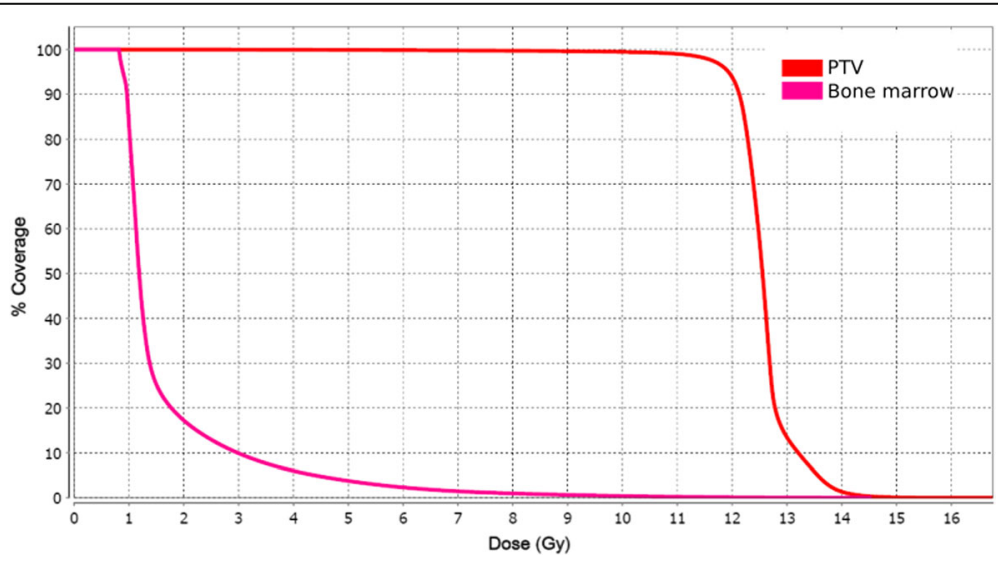

Fig. 2 Dose-volume histogram for patient 1 treated with TSHT for mycosis fungoides 
Table 1 Patient 1 dose values

\begin{tabular}{|c|c|c|c|c|}
\hline & Mean & $\mathrm{V} 10$ & V5 & V3 \\
\hline Bone marrow total (arms not included) & $1.66 \mathrm{~Gy}$ & $0.41 \%$ & $3.72 \%$ & $9.81 \%$ \\
\hline Bone marrow total (arms included) & $2.62 \mathrm{~Gy}$ & $7.97 \%$ & $13.45 \%$ & $19.03 \%$ \\
\hline Lumbar & $1.51 \mathrm{~Gy}$ & $0.97 \%$ & $3.15 \%$ & $7.65 \%$ \\
\hline Dorsal & $1.95 \mathrm{~Gy}$ & $0.49 \%$ & $3.76 \%$ & $11.19 \%$ \\
\hline Pelvic & $1.49 \mathrm{~Gy}$ & $0.32 \%$ & $3.16 \%$ & $7.55 \%$ \\
\hline Femurs & $1.86 \mathrm{~Gy}$ & $0.12 \%$ & $4.86 \%$ & $14.09 \%$ \\
\hline Arms & $11.18 \mathrm{~Gy}$ & $74.54 \%$ & $98.84 \%$ & $100 \%$ \\
\hline
\end{tabular}

of treatment completion, the patient noted significant improvement of her diffuse erythroderma. Despite radiotherapy she had progressive disease on her right thigh several weeks later and began treatment with chlorambucil and low dose prednisone. She continued chlorambucil until she was hospitalized and ultimately passed away secondary to stroke several months after treatment.

The second patient was a 29-year-old Caucasian female with stage I (T2 N0 M0 B0) mycosis fungoides. She had previous treatment with topical steroids, methotrexate, interferon, mycophenolate, and cyclosporine. She was on photochemotherapy with interferon alfa-2b at the time of presentation. At that time, she was treated with photons to active sites of disease with a dose of 20 Gy in 10 fractions to the scalp, buttocks, neck, and axillae, and a dose of 10 Gy to the back in 5 fractions. The patient experienced improvement in disease burden. However, the patient also experienced myelosuppression beginning 2 weeks after this treatment with grade 3 leukopenia, grade 1 anemia, and grade 4 thrombocytopenia.

She then presented again to the radiation oncology clinic after progression of disease approximately 17 months from her previous radiotherapy treatment. She was being treated with interferon alfa- 2 and methotrexate. She was then treated to a dose of 12 Gy in 6 fractions with daily treatments using TSHT with sparing of the hands and feet. The patient underwent simulation and treatment with a facemask and body fix with arms down The upper half and lower half of the body were treated separately with each hemibody receiving 6 fractions of 2 Gy. The dose distribution and dose-volume histogram are shown in Figs. 3 and 4. Bone marrow mean dose was 2.3 Gy with $\mathrm{V} 10=4.28 \%$. As with the first patient, the bone marrow contour sum includes the entire spine, pelvis and femurs. Dose values to other bony sites have been included for additional reference in Table 2.

Initially the patient had a good response to therapy with many of the cutaneous lesions resolving, however her disease rapidly progressed 2 months following treatment. Bone marrow suppression occurred 3 weeks after treatment with grade 1 anemia, grade 3 leukopenia, and grade 4 thrombocytopenia. The patient did not report any bleeding events, however red blood cell and platelet transfusions were necessary before the patient's myelosuppression eventually resolved 6 months after the end of treatment.
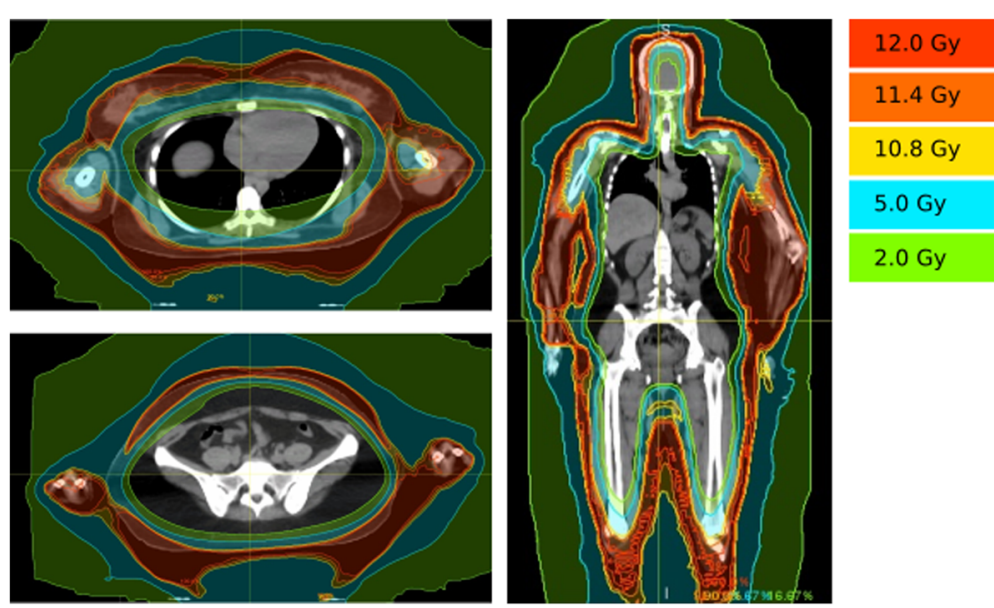

Fig. 3 Dose distributions for patient 2 treated with TSHT for mycosis fungoides. Note that the hands and feet of this patient were spared treatment 


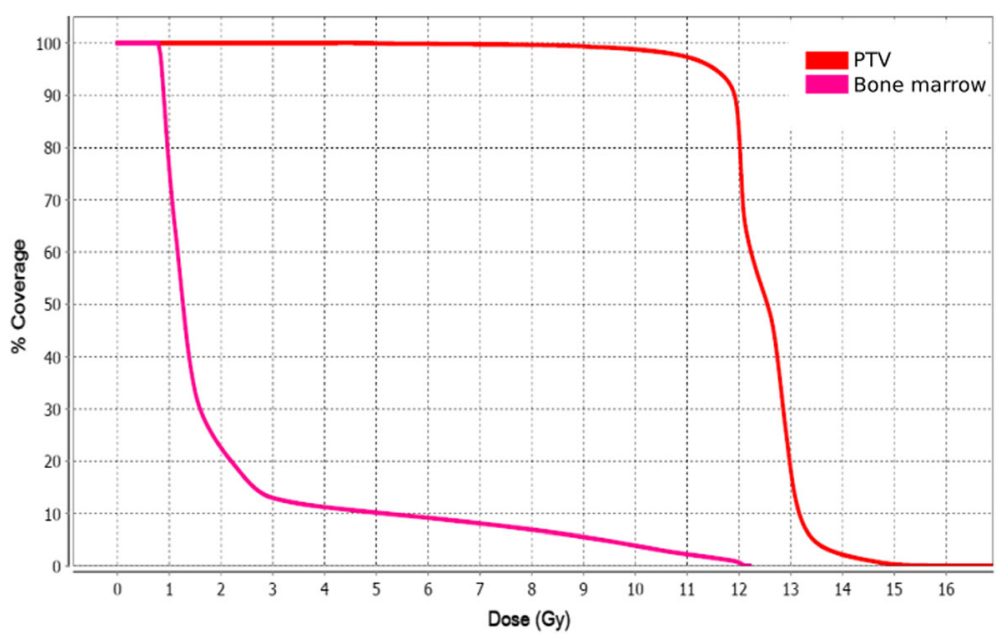

Fig. 4 Dose-volume histogram for patient 2 treated with TSHT for mycosis fungoides

\section{Discussion and conclusions}

Both patients treated with TSHT at our institution experienced significant bone marrow toxicity. Even at a prescription dose of $12 \mathrm{~Gy}$, these patients experienced grade 4 myelosuppression. Their platelet nadir occurred 2 and 3 weeks after treatment ended. This was similar to the 2-week platelet nadir Carabell et al. experienced in patients treated with total body irradiation (TBI) to a dose 1.5 Gy for advanced non-Hodgkin lymphoma [7]. Similar bone marrow toxicities have been reported when using TBI to a dose of 1.5 Gy with concurrent lonidamine to treat patients with favorable B-cell neoplasms as well [8]. Our results show similar rates of myelosuppression as patients treated using TSHT at a higher prescription dose of $30 \mathrm{~Gy}$ in the series by Hsieh et al. [9]. However, in the prior report, the mean bone marrow dose varied between 4 to $9 \mathrm{~Gy}$. In our two patients, the mean bone marrow doses were significantly lower at 2 . 3 Gy and 1.66 Gy.

In a Japanese case series, 3 patients were treated for mycosis fungoides using TSHT to a dose of 10 Gy and myelosuppression occurred in 2 patients [10]. One of the patients experienced grade 4 myelosuppression which later required blood transfusion. This study is comparable to our own, as prescription doses (12 Gy vs 10 Gy) were similar. In addition, their mean bone marrow dose of 2.27 Gy was comparable to our patients. Fractionation similar to our first patient (12 Gy in 8 fractions) has been shown to result in rare grade 2 and grade 3 toxicities without myelosuppression when using TSEBT instead of TSHT $[11,12]$.

The question remains as to why our patients and the patient from the previously mentioned case study experienced severe myelosuppression with such low mean bone marrow dose. One explanation is that our treatment planning software may have not have accurately simulated the actual bone marrow dosage. Another possibility is that the planning parameter of a mean bone marrow dose $<2$ Gy is not a strict or predictive enough treatment planning constraint for total skin treatments. Thrombocytopenia experienced in these two patients may be secondary to significant volume of low dose irradiation to the bone marrow. A total body exposure of 2.5-5 Gy results in hematopoietic syndrome. This occurs secondary to destruction of mitotically active precursor cells approximated 3-4 weeks after exposure. In these patients, mean doses to the bone marrow did approach 2 Gy and likely led to similar biological effects and the resultant thrombocytopenia.

Table 2 Patient 2 dose values

\begin{tabular}{|c|c|c|c|c|}
\hline & Mean & V10 & V5 & V3 \\
\hline Bone marrow total (arms not included) & $2.3 \mathrm{~Gy}$ & $4.28 \%$ & $10.11 \%$ & $12.93 \%$ \\
\hline Bone marrow total (arms included) & $3.56 \mathrm{~Gy}$ & $13.76 \%$ & $24.12 \%$ & $27.49 \%$ \\
\hline Lumbar & $1.18 \mathrm{~Gy}$ & 0 & 0 & $0.76 \%$ \\
\hline Dorsal & $1.87 \mathrm{~Gy}$ & 0 & $0.12 \%$ & $2.61 \%$ \\
\hline Pelvic & $1.13 \mathrm{~Gy}$ & 0 & $0.03 \%$ & $0.71 \%$ \\
\hline Femurs & $4.53 \mathrm{~Gy}$ & $14.31 \%$ & $37.38 \%$ & $44.77 \%$ \\
\hline Arms & 10.41 Gy & $62.97 \%$ & $93.77 \%$ & $100 \%$ \\
\hline
\end{tabular}


One method that may decrease internal dose would be using a bolus to bring the dose closer to the skin surface. This was done in an anthropomorphic phantom substitute study by Lin et al. in which TSHT was used to produce a dose of at least 26 Gy to a depth of $4 \mathrm{~mm}$ of the truncal skin in conjunction with a $3 \mathrm{~mm}$ neoprene diving suit utilized as bolus [13]. This plan resulted in a simulated mean dose to OARs of 9.7 cGy per fraction during the 36 Gy treatment course of 36 fractions as compared to approximately $1-4 \mathrm{cGy} / \mathrm{fx}$ with a rival TSEBT plan. However, they defined the OARs to be heart, lung, liver, kidney, spleen, intestine, and rectum, while bone marrow was not included. Hsieh et al. also used a $3 \mathrm{~mm}$ neoprene diving suit to act as bolus in their actual patient treatment setup, however their patient still experienced grade 4 myelosuppression.

Another concern with patients receiving significant bone marrow radiation dose would be secondary malignancies such as leukemia and myelodysplastic syndrome. This has been reported in patients receiving a mean bone marrow dose of 5.2 Gy when treated with total body irradiation and chemotherapy for non-Hodgkin lymphoma [14].

Disease response was moderate in our two treated cases with each experiencing a partial response immediately following treatment but soon after relapsing. Prior success had been shown with TSEBT to a dose of $12 \mathrm{~Gy}$ with a complete response in $88 \%$, but often repeat total skin treatment may be needed at these low doses [7].

Although TSHT allows for improvements in dose homogeneity when compared to TSEBT, these cases illustrate the need for further research on the safety of TSHT, specifically in the case of bone marrow toxicity. We attempted to avoid bone marrow toxicity by limiting the mean dose to $2 \mathrm{~Gy}$. Yet, significant myelosuppression was still encountered. Caution should be utilized when using TSHT as a therapy for mycosis fungoides. Further, patients will likely benefit from enrollment on various approaches to total skin especially in the context of TSHT. Patients who have been previously treated or are currently being treated with chemotherapy that have a low hematopoietic reserve may be especially at risk. Currently, clinical trials are underway to examine patients who will receive TSHT for treatment resistant cutaneous lymphoma [15]. These results will be a welcome addition to the literature and may help clarify safety and tolerability of this treatment approach.

\section{Abbreviations}

HT: Helical tomotherapy; OAR: Organs at risk; PRBC: Packed red blood cell; TBI: Total body irradiation; TSEBT: Total skin electron beam therapy;

TSHT: Total skin helical tomotherapy

\section{Authors' contributions}

All authors contributed to drafting the manuscript and approved the final manuscript.
Consent for publication

Patients signed consent prior to treatment being initiated and data being collected.

\section{Competing interests}

The authors declare that they have no competing interests.

\section{Publisher's Note}

Springer Nature remains neutral with regard to jurisdictional claims in published maps and institutional affiliations.

\section{Author details}

${ }^{1}$ Michigan State University College of Human Medicine, 418 W. Magnetic Street, Marquette, Ml 49855, USA. ${ }^{2}$ Department of Human Oncology, University of Wisconsin, Madison, WI, USA.

Received: 20 November 2017 Accepted: 2 April 2018

Published online: 13 April 2018

\section{References}

1. De Moraes FY, HDA C, Hanna SA, JLF DS, Marta GN. Literature review of clinical results of total skin electron irradiation (TSEBT) of mycosis fungoides in adults. Rep Pract Oncol Radiother. 2014;19(2):92-8.

2. Guerts M, Bayliss A, Thapa B, Nelson G. Total skin tomotherapy for treatment of mycosis fungoides. 2014. https://www.researchgate.net/publication/ 267453606_Total_Skin_TomoTherapy_for_Treatment_of_Mycosis_ Fungoides. Accessed 16 Aug 2017.

3. Weaver RD, Gerbi BJ, Dusenbery KE. Evaluation of dose variation during total skin electron irradiation using thermoluminescent dosimeters. Int J Radiat Oncol Biol Phys. 1995;33(2):475-8.

4. Wu JM, Leung SW, Wang CJ, Chui CS. Lying-on position of total skin electron therapy. Int J Radiat Oncol Biol Phys. 1997;39(2):521-8.

5. Elsayad K, Susek KH, Eich HT. Total skin electron beam therapy as part of multimodal treatment strategies for primary cutaneous T-cell lymphoma. Oncol Res Treat. 2017:40:244-52.

6. Hoppe RT, Harrison C, Tavallaee M, Bashey S, Sundram U, Li S, et al. Low-dose total skin electron beam therapy as an effective modality to reduce disease burden in patients with mycosis fungoides: results of a pooled analysis from 3 phase-II clinical trials. J Am Acad Dermatol. 2014;72(2):286-92.

7. Carabell SC, Chaffey JT, Rosenthal DS, Moloney WC, Hellman S. Results of total body irradiation in the treatment of advanced non-hodgkin's lymphomas. Cancer. 1979;43(3):994-1000.

8. Robins HI. Combined modality clinical trials for favorable B-cell neoplasms: lonidamine plus whole body hyperthermia and/or total body irradiation. Semin Oncol. 1991;18(2):23-7.

9. Hsieh $\mathrm{CH}$, Shueng PW, Lin SC, Tien HJ, Shiau AC, Chou YH, et al. Helical irradiation of the total skin with dose painting to replace total skin electron beam therapy for therapy-refractory cutaneous CD4+ T-cell lymphoma. Biomed Res Int. 2013; https://doi.org/10.1155/2013/717589.

10. Okuma K, Haga A, Imae T, Takenaka R, Sugaya M, Nakagawa K. Total skin irradiation using helical tomotherapy: a novel experience and report of three cases. Radiother Oncol. 2016;119(1):676.

11. Kroeger K, Elsayad K, Moustakis C, et al. Low-dose total skin electron beam therapy for cutaneous lymphoma. Strahlenther Onkol. 2017;193:1024-30.

12. Morris S, Scarisbrick J, Frew J, et al. The results of low-dose total skin electron beam radiation therapy (TSEB) in patients with mycosis fungoides from the UK cutaneous lymphoma group. Int J Radiat Oncol Biol Phys. 2017;

13. Lin CT, Shiau AC, Tien HJ, Yeh HP, Shueng PW, Hsieh CH. An attempted substitute study of total skin electron therapy technique by using helical photon tomotherapy with helical irradiation of the total skin treatment: a phantom result. Biomed Res Int. 2013; https://doi.org/10.1155/2013/108794.

14. Travis LB, Weeks J, Curtis RE, Chaffey JT, Stovall M, Banks PM, et al. Leukemia following low-dose total body irradiation and chemotherapy for nonHodgkin's lymphoma. J Clin Oncol. 1996;14(2):565-71.

15. Helical irradiation of total skin (HITS) for cutaneous lymphoma. 2017. Retrieved from https://clinicaltrials.gov/ct2/show/NCT02039895 (Identification No. NCT02039895). 